196 RUNNING PATTERN ASYMMETRY EVALUATION AFTER ANTERIOR CRUCIATE LIGAMENT RECONSTRUCTION COULD BE A WAY TO DETECT RE-INJURIES

1,2 Alexandre Rambaud, 1,3Thomas Neri, ${ }^{3}$ Jean-Benoit Morin 1,3 Remi Philippot, ${ }^{1}$ Jeremy Rossi, ${ }^{5}$ Pierre Samozino, ${ }^{1,6}$ Pascal Edouard. 'Univ Lyon, UJM-Saint-Etienne, Laboratoire Interuniversitaire de Biologie de la Motricité, EA 7424, F-42023, Saint-Etienne, France; ${ }^{2}$ SFMKS Lab, Pierrefitte-sur-Seine, France; ${ }^{3}$ Department of Orthopedic Surgery, University Hospital Center of Saint-Etienne, Saint-Etienne, France; ${ }^{5}$ Univ Savoie Mont Blanc, Laboratoire Interuniversitaire de Biologie de la Motricité, EA 7424, F-73000, Chambéry, France; ${ }^{6}$ Department of Clinical and Exercise Physiology, Sports Medicine Unit, University Hospital of Saint-Etienne, Faculty of Medicine, Saint-Etienne, France

\subsection{6/bjsports-2021-IOC. 181}

Background A test battery is recommended after anterior cruciate ligament reconstruction (ACLR) to help decision-making of return to sport (RTS). Running is important in RTS continuum, but it is currently not included in the usual test batteries. Could running pattern asymmetry, which can be observed after ACLR, be considered as a risk factor of ACL re-injuries?

Objective To analyse the interest of adding running pattern evaluation in test battery after ACLR.

Design Prospective cohort study.

Setting Patient with ACLR with Tegner score $>6$ and Marx Scale score $>8$ before first ACL injury.

Patients (or Participants) 21 patients (12 women) with primary ACLR (graft type: Patellar $(n=4)$ or Hamstring tendon $(n=17))$ without major chondral and/or meniscal lesion.

Interventions (or Assessment of Risk Factors) Running pattern evaluation was performed on a motorised instrumented treadmill in addition to recommended tests (questionnaires, knee laxity, Hop Tests and isokinetic evaluation) at 6 months after ACLR. ACL re-injuries were prospectively collected during the two years after ACLR, and comparisons between parameters were performed between ACLR patients who presented or not an ACL re-injury

Main Outcome Measurements Limb symmetry Index (LSI=operated leg/healthy leg x100) were calculated for running variables (Stride Length, Loading Rate, Leg Stiffness) and other parameters of test battery. Means were compared between groups.

Results Five patients had a re-injury ( $3 \mathrm{~W} / 2 \mathrm{M})$, without significant differences in baseline characteristics (sport level, training frequency) with un-re-injured patients. For running variables, there was a significant difference for LSIs of Stride Length (Re-injury group $95.8 \pm 2.3 \%$ vs $99.0 \% \pm 1.8 \%$, $\mathrm{p}=0.003)$, Loading Rate $(86.9 \pm 13.3 \%$ vs $98.3 \pm 9.6 \%$, $\mathrm{p}=0.025)$, and Leg Stiffness $(112.6 \pm 7.3 \%$ vs $103.7 \pm 8.0 \%$, $\mathrm{p}=0.035$ ), while there were no statistical differences for LSIs of other parameters of test battery.

Conclusions As greater running pattern asymmetry was reported in patients with ACL re-injury, this approach could be of interest for secondary prevention.

\section{RESPONSIVENESS OF THE ANTERIOR CRUCIATE LIGAMENT - RETURN TO SPORTS AFTER INJURY (ACL- RSI) AND INJURY - PSYCHOLOGICAL READINESS TO RETURN TO SPORT (I-PRRS) SCALES}

Anton Slagers, Johannes Zwerver. University of Groningen, University Medical Center Groningen, Center for Rehabilitation, Department of Rehabilitation Medicine, Groningen, Netherlands

10.1136/bjsports-2021-IOC. 182
Background Both physical and psychological readiness are important for a successful return to sport (RTS) and secondary prevention. The ACL-Return to Sport after Injury (ACLRSI) and Injury-Psychological Readiness to Return to Sport (IPRRS) scales were developed to assess psychological factors associated with RTS. Validity and reliability have been determined but responsiveness of both scales has not been examined yet.

Objective To investigate the responsiveness of the Dutch ACLRSI and I-PRRS scales.

Design Prospective, cohort study.

Setting Patients from a university and regional hospital.

Patients Seventy athletes with ACL reconstruction 3-9 month previous to the start of the study completed both scales twice two months apart, plus a Global Rating of Change (GRC) questionnaire.

Main Outcome Measurements Distribution and logistic regression-based methods were used to study responsiveness.

Results The Standardized Response Mean (SRM) for the ACLRSI was 0.3 and for the I-PRRS 0.1. The minimally important change (MIC) for ACL-RSI was 2.6 and for the I-PRRS 0.9. Since the smallest detectable change (SDC) was larger than MIC in individual patients, it does not seem possible to distinguish minimally important changes from measurement error in individual patients with either scale. At group level responsiveness of both scales seemed sufficient.

Conclusions In individual patients the responsiveness of the ACL-RSI and I-PRRS scales seems to be insufficient to detect changes in confidence over time with regard to return to sport after ACLR. Neither scale is able to distinguish minimally important changes from measurement error in individual patients. The scales are therefore less suitable for monitoring the effectiveness of individual interventions and to judge whether a patient has reached a change of importance. At the group level responsiveness seems sufficient, so the two scales can be used to investigate the effectiveness of an intervention at the group level.

\section{CHALLENGING ACL RECONSTRUCTED ATHLETES AND THEIR SENSORIMOTOR SYSTEM AT RETURN-TO-SPORT: A VITAL STEP TOWARDS EXPOSING THE ROOTS OF THEIR NEUROMUSCULAR DEFICITS}

${ }^{1}$ Annemie Smeets, 'Sabine Verschueren, ${ }^{1}$ Filip Staes, ${ }^{2}$ Steven Claes, ${ }^{3}$ Hilde Vandenneucker, 'Jos Vanrenterghem. 'Musculoskeletal Rehabilitation Research Group, Department of Rehabilitation Sciences and Physiotherapy, Faculty of Kinesiology and Rehabilitation Sciences, KU Leuven, Leuven, Belgium; ' ${ }^{2}$ Department of Orthopedic Surgery, AZ Herentals Hospital, Herentals, Belgium; ${ }^{3}$ Department of Orthopedics, University Hospitals Leuven, Leuven, Belgium

\subsection{6/bjsports-2021-IOC.183}

Background Evidence increasingly suggests that neuromuscular alterations in ACL reconstructed (ACLR) patients are rooted in neurocognitive and proprioceptive deficits.

Objective The aim of this study was to reveal how neurocognitive and proprioceptive deficits determine neuromuscular control alterations seen in ACLR athletes.

Design Cross-sectional study.

Setting Athletes who return to a cutting or pivoting sport after an ACL reconstruction.

Patients 20 athletes who had an ACL reconstruction and were cleared by the surgeon/physiotherapist to return to sport (RTS), were tested at time of RTS. A control group of 20 
uninjured athletes, matched for gender and type of sport, was included.

Assessment of Risk Factors All participants performed a stepping-down task under 4 conditions: 1 . no additional challenges (NORM), 2. whilst performing a cognitive dual-task (DUAL), 3. whilst undergoing unpredictable surface perturbations (PERT), 4. whilst performing a cognitive dual-task + undergoing unpredictable surface perturbations.

Main Outcome Measurements Muscle activations of the vastus medialis (VM), vastus lateralis, hamstrings medialis (HM), hamstrings lateralis were recorded with surface EMG. Integrals were calculated over the landing period (50-250 ms after initial contact) and normalized to maximal voluntary contractions.

Results The ACLR athletes showed an almost unadjusted strategy of increased HM (6.7\% (ACL); 3.9\% (control), $\mathrm{F}=5.07, \mathrm{p}=0.031)$ and decreased $\mathrm{VM}$ activation across all tasks (6.8\% (ACL); $12.9 \%$ (control), $\mathrm{F}=8.52, \mathrm{p}=0.006$ ), whilst the control group had a clear increase in HM activation (3.2\% (NORM); 5.6\% (PERT), t=4.06, $\mathrm{p}=0.001)$ and $\mathrm{VM}$ activation $(9.0 \% \quad(\mathrm{NORM}) ; 16.9 \% \quad(\mathrm{PERT}), \mathrm{t}=3.81$, $\mathrm{p}=0.001$ ) when unpredictable perturbations occurred. However, within the ACLR athletes HM activation decreased when a dual task was added (7.3\% (NORM); 4.7\% (DUAL), $\mathrm{t}=3.4, \mathrm{p}=0.003)$.

Conclusions It seems that the neuromuscular strategy of the ACLR athlete is an overprotective strategy to improve knee stability. They use this strategy in every situation, potentially to compensate for the altered proprioceptive input. This overprotective strategy is jeopardized under cognitively challenging circumstances, confirming that underlying neurocognitive limitations contribute to altered neuromuscular control in ACLR athletes.

\section{GENDER DIFFERENCES IN LANDING MECHANICS AFTER ANTERIOR CRUCIATE LIGAMENT RECONSTRUCTION}

${ }^{1}$ Ahmad Alanazi, ${ }^{2}$ Faisal Al-Enezi, ${ }^{3}$ Mishal Aldaihan, ${ }^{4}$ Hamad Al Amer, ${ }^{5}$ Alexis Ortiz. ${ }^{1}$ Department of Physical Therapy. Majmaah University, Majmaah, Saudi Arabia; ${ }^{2}$ Therapeutic Deputyship, Ministry of Health, Riyadh, Saudi Arabia; ${ }^{3}$ Department of Rehabilitation Health Sciences, King Saud University, Riyadh, Saudi Arabia; ${ }^{4}$ Department of Physical Therapy, University of Tabuk, Tabuk, Saudi Arabia; ${ }^{5}$ UT Health San Antonio, San Antonio, USA

\subsection{6/bjsports-2021-IOC.184}

Background Studies have reported that females and males exhibit different landing techniques. However, few studies have examined the effect of gender on landing technique in athletes following ACLR.

Objective To compare landing technique between females and males following ACLR during landing from heading-jump (LHJ) and landing from long-jump (LLJ).

Design Cross-sectional study.

Setting Biomechanical laboratory.

Patients (or Participants) Eight female and 8 male recreational athletes.

Interventions (or Assessment of Risk Factors) LHJ included jumping forward to head a soccer ball and landing on the force plates, whereas LLJ included jumping forward and landing on the force plates.

Main Outcome Measurements A $2 \times 2$ ANOVA (gender $\times$ landing) was performed to evaluate kinematics, kinetics, and electromyography data.
Results A significant interaction was found only for knee flexion angles $(F 1,14=12.67, p=0.003)$. Pairwise comparisons showed that males landed with decreased knee flexion compared with females during LLJ $(\mathrm{p}=0.01)$. LHJ showed decreased knee flexion compared with LLJ in females $(p<$ $0.001)$ and males $(p=0.001)$. Significant main effects of landing were found. LHJ showed decreased hip flexion angles $(\mathrm{F} 1,14=71.07, \mathrm{p}<0.001)$, decreased knee flexion angles $(\mathrm{F} 1,14=95.17, \mathrm{p}<0.001)$, decreased knee extension moments $(\mathrm{F} 1,14=20.12, \mathrm{p}=0.001)$, and decreased plantarflexion moments $(\mathrm{F} 1,14=34.71, \mathrm{p}<0.001)$. Also, a significant main effect of gender for hip flexion was found showing that males landed with decreased hip flexion angles $(\mathrm{F} 1,14=$ 7.17, $\mathrm{p}=0.01$ ).

Conclusions LHJ showed greater injury predisposing factors compared with LLJ. Females and males following ACLR showed nearly similar landing biomechanics. However, males landed with smaller hip and knee flexion angles (stiff-landing); therefore, preventative training programs may focus on improving the use of hip and knee joints (soft-landing) during landing to decrease the risk of consequent injuries in males following ACLR.

\section{RELATIONSHIP BETWEEN POLE VAULT BIOMECHANICS AND PREVIOUS INJURIES}

1,2,3,4,5 Pascal Edouard, ${ }^{6}$ Hervé Sanchez, ${ }^{3}$ Cyprien Bourrilhon, ${ }^{6}$ Sébastien Homo 7,8 Julien Frère, ${ }^{9,10}$ Johan Cassirame. ${ }^{1}$ Inter-university Laboratory of Human Movement Science (LIBM EA 7424), University of Lyon, University Jean Monnet, Saint-Etienne, France; ${ }^{2}$ Department of Clinical and Exercise Physiology, Sports Medicine Unit, University Hospital of Saint-Etienne, Faculty of Medicine, Saint-Etienne, France; ${ }^{3}$ Medical Commission, French Athletics Federation (FFA), Paris, France; ${ }^{4}$ Swiss Olympic Medical center, Centre de médecine du sport, Division de médecine physique et réadaptation, Centre Hospitalier Universitaire Vaudois, Lausanne, Switzerland; ${ }^{5}$ European Athletics Medical and Anti Doping Commission, European Athletics Association (EAA), Lausanne, Switzerland; ${ }^{6}$ French Athletics Federation (FFA), FIR division, Paris, France; ' Université de Lorraine, Faculty of Medicine, Laboratory " Développement, Adaptation et Handicap » (EA 3450), Vandoeuvre-lès-Nancy, France; ${ }^{8}$ Université de Lorraine, Faculty of Sports Sciences, Villers-lès-Nancy, France; ${ }^{9} E A$ 4660, Culture, Sport, Health and Society Department and Exercise Performance, Health, Innovation platform, University of Bourgogne France Comté, Besançon, France; ${ }^{10}$ EA 7507, Laboratoire Performance, Santé, Métrologie, Société, Reims, France

\subsection{6/bjsports-2021-IOC.185}

Background During pole vault, a high level of energy is transferred from horizontal speed of athlete to the pole. Several ways of performing the pole vault exist, and could be associated with specific injury risk.

Objective To analyse the potential association between the biomechanical patterns of pole vault and the history of injuries.

Design Retrospective study.

Setting National elite indoor championship and youth national indoor championship (U17 and U20) of pole vault (athletics)

Participants $62(70.5 \%$ of the eligible population participating in the championships) healthy national level male and female pole vaulters.

Main Outcome Measurements We prospectively collected the pole vault biomechanical data of the run-up and take-off phases of the vault, and we retrospectively collected the history of injuries during the 12 preceding months through an online questionnaire.

Results A total of $62 \quad(70.5 \%$ of those participating in the championships) pole vaulters accepted to participated in this study, and benefited from pole vault biomechanical and injury 